\title{
BIBECHANA
}

ISSN 2091-0762 (Print), 2382-5340 (Online)

Journal homepage: http://nepjol.info/index.php/BIBECHANA

Publisher: Department of Physics, Mahendra Morang A.M. Campus, TU, Biratnagar, Nepal

\section{Spatial orientations of angular momentum vectors of galaxies in Supercluster S [173+014+0082]}

\author{
Janak Ratna Malla ${ }^{\mathbf{*}}$, Walter Saurer ${ }^{2}$, Binil Aryal ${ }^{\mathbf{1}}$ \\ ${ }^{1}$ Central Department of Physics, Kirtipur, Kathmandu \\ ${ }^{2}$ Institute of Astro-particle Physics, Innsbruck University, Austria \\ *Email: janak_malla@yahoo.com
}

\section{Article Information:}

Received: May 28, 2020

Accepted: June 16, 2020

Keywords:

SDSS

Supercluser

Galaxy

Redshift

Intrinsic flatness

\begin{abstract}
The spin vector orientation of 1302 SDSS (Sloan Digital Sky Survey) galaxies in Supercluster S[173+014+0082] having redshift 0.076 to 0.091 has been analysed. The positions, position angles and inclination angles of galaxies are used to convert two-dimensional observed parameters into three-dimensional angular momentum vectors of the galaxy using the 'position angle-inclination' method. The expected isotropic distribution curves are determined performing numerical simulation by generating 107 virtual galaxies. The observed distribution is compared with the expected isotropic distribution curves using three statistical tools namely Chi-square test, auto-correlation test and Fourier test. Redshift map is studied and found that the distributions fit with the Gaussian. No preferred alignment of angular momentum vectors is noticed, supporting Hierarchy model of galaxy formation.
\end{abstract}

DOI: https://doi.org/10.3126/bibechana.v18i1.29165

This work is licensed under the Creative Commons CC BY-NC License. https://creativecommons.org/licenses/by-nc/4.0/

\section{Introduction}

During the path of history different ideas had been recommended describing the starting place of the universe. The most persuasive model is the standard Big Bang model, which remains valid. This model shows the creation technique of the universe began with an enlargement 20 billion years ago. Nowadays, the big Bang model is believed to be the beginning of space and time. The tiny, hot $\left(10^{12} \mathrm{~K}\right)$ universe started out to cool and fundamental symmetry damaged down. The breaking of this symmetry results in the inflation of the universe exponentially. Within a fraction of second, after the Big Bang, the procedure of Nucleosynthesis, baryongenesis \& leptogenesis took place. After this procedure the universe entered into the matter dominated era. During dark epoch, the stars and galaxies are assumed to be formed. Today, how matter is distributed in the universe is the crucial field of study in cosmology and astronomy.

The mutual attraction between the galaxies due to the gravitational force leads to the formation of clusters. Depending upon the number of galaxies 
inside the cluster, they're labeled as poor and rich clusters. In case of poor clusters the force of gravity is weak to keep them tightly due to lower mass of the galaxy. Thus they seem irregular in shape. Our Milky Way is part of a poor cluster referred to as a local group which includes 54 galaxies [1]. The Virgo cluster is an example of a moderately rich cluster that is closest to us and the coma cluster is a rich cluster, has thousands of spiral and elliptical galaxies. The clustering technique of galaxy clusters lead to the formation of large scale structures known as superclusters. Thus they're collections of galaxy clusters, with $20-100 \mathrm{~h}^{-1} \mathrm{Mpc}$ [2]. The scientist assumed that the variety of superclusters in the universe is set 10 million and it occupies the $10 \%$ area of the universe. As it contains organizations and clusters of galaxies, it provides the strong proof that galaxies inside the universe are not distributed uniformly. Abell catalogue is the pioneer work in the study of superclusters. It was proposed by George Abell in 1958 and he called them second order cluster or clusters of clusters [3]. The study of superclusters highly benefited from the $2 \mathrm{dF}$ Galaxy Redshift Survey (2dFGRS) and the Sloan Digital Sky Survey (SDSS) [4]. Currently Sloan Digital Sky Survey (SDSS) is taking data from the deep sky. Our work is based totally on the data provided by SDSS-DR7.

As suggested by Weizscker \& Gamow [5, 6], the observe of origin of angular momentum of galaxies might be the clue for the physical properties of the initial situations of galaxies. Thus, the observed rotation of galaxy is critical to the cosmology and subsequently it is essential to recognize how they obtained their angular momentum. Pebbles [7] put forward detail calculations of the acquisition of angular momentum in early stage of proto-galactic evolution. He argued that, the gravitational interplay of quadrapole moment of the system with tidal field of the matter causes transformation of angular momentum to the developing proto-galaxy. The transformation of angular momentum between rotation and orbital motion is a outcome of the gravitational instability [8]. Peebles assumed that, as soon as the primeval plasma has recombined and decoupled from the primeval fireball radiation, gravitation appears to be the dominant force transferring the angular momentum to a developing proto-galaxy.

On the basis of orientation of angular momentum vectors of galaxies one can determine the evolution of large scale structure, i.e., Supercluster. The alignment of rotational axes of galaxies in the Supercluster provides us information about the formation process of galaxies in early epoch [9]. There had been contradictory theoretical models predicting various galaxy cluster formation. The Hierarchy model [7] predicts random orientation of angular momentum vectors. The Pancake model [10] predicts the parallel orientation whereas the primordial vorticity model [11] says angular momentum vector distributed perpendicular to the cluster plane. We are interested to study the evolution of galaxies in the Supercluster by analyzing their preferred alignments.

\section{Materials and Method \\ Database}

The analyzed data set has been recorded by Sloan Digital Sky Survey (SDSS). With the collaboration of Prof. Walter Saurer's group at the Institute of Astro-particle Physics, Innsbruck University, Austria, we obtained the set of SDSS (7th Data release) data. This Supercluster S $[173+014+$ 0082] contains a total number of 1302 galaxies having redshift ranges from 0.076 to 0.091 . In this work, r-filter is used to study the spin vector orientation of the galaxies. In order to calculate inclination angle first of all we used intrinsic flatness $\left(q^{*}\right)=0.2$ (Holmberg, [12]) and, then 0.13 for those galaxies whose inclination angle was undetermined from the previous value of $q^{*}$. In this process nine galaxies were excluded whose intrinsic flatness is less than 0.13 . Hence, finally there are 1293 galaxies in the data set whose position (right ascension and declination), position angle and inclination are known. 


\section{Godlowskian transformation}

The two dimensional parameters such as position, position angle and diameter can be transformed into 3 dimensional parameters using the method proposed by Flin and Godlowski [13] and polar $(\theta)$ and azimuthal $(\phi)$ angle can be calculated for each galaxy. The polar angle $(\theta)$ is the angle between galactic spin vector $(\mathrm{SV})$ and reference plane, and azimuthal angle $(\phi)$ is the angle between projection of a galactic spin vector to the reference plane and $\mathrm{X}$-axis. These angles are given below.

$\sin \theta=-\cos i \sin \alpha \pm \sin i \sin p \cos \delta$

$\sin \phi=(\cos \theta)^{-1}[-\cos i \cos \delta \sin \alpha+$ $\sin i(\mp \sin p \sin \delta \sin \alpha \mp \cos p \cos \alpha)]$

Here, i, $\delta, \alpha$, and $\rho$ represent the inclination angle, declination, right ascension and position angles respectively and $\mp$ and \pm indicate the two possible solution.

The inclination angle (i) defined the angle between normal to the galaxy plane and line of sight of the observer. This is given by following relation:

$$
\cos ^{2} i=\frac{\left(\frac{b}{a}\right)^{2}-q^{* 2}}{1-q^{* 2}}
$$

Here b/a is axial ratio and $\mathrm{q}^{*}$ represents the intrinsic flatness factor of the galaxy. For the disk galaxies its value depends upon the morphological type. Holmberg [12] showed that the values of $\mathrm{q}^{*}$ varies from 0.083 for $\mathrm{Sd}$ spirals to 0.3 for elliptical. At first, $\mathrm{q}^{*}=0.20$ for morphologically unidentified galaxies which leads to the removal of a certain number of galaxies that have axial ratio (b/a) is less than 0.20 . Then, we used $q^{*}=0.13$ for those galaxies that have $b / a<0.20$.

\section{Method of analysis}

Aryal \& Saurer [14], proposed a method for the removal the selection effects and the expected isotropic distribution curves $(\theta$ and $\phi)$ are determined using the numerical simulation. For this, a true spatial distribution of the galaxy rotation axis is assumed to be isotropic. Then, due to the projection effects, $i$ can be distributed $\propto \sin \mathrm{i}$, latitude can be distributed $\propto \cos \mathrm{B}$, the variables longitude $(\alpha)$ and position angle (p) can be distributed randomly and formulae (1) and (2) can be used to simulate (numerically) the corresponding distribution of $\theta$ and $\phi$. At first, the isotropic distribution curves are based on simulations including $10^{7}$ virtual galaxies Aryal \& Saurer [14]. We use these numbers to make an input file and the predicted distribution by using a running simulation in MATLAB 15.0.

Finally, the observed and expected distributions are compared with the help of appropriate statistical tests. In order to use ORIGIN 5.0 to determine the all sky distribution of galaxies where as ORIGIN 8.0 used for the redshift distribution of galaxy.

\section{Results and Discussion}

All sky distribution is the pictorial representation of distribution of galaxies within a certain range of right ascension and declination in the sky. The all sky distribution plot for our Supercluster $S$ [173++014+0082] is shown in Fig. 1. The diagram shows that galaxies are distributed within the range of right ascension from $170^{\circ}$ to $181^{\circ}$ and declination from $6^{0}$ to $18^{0}$.

Figure 1 shows that galaxies are distributed inhomogenously. To study the preferred alignment of each galaxy, we plotted the various parameters of galaxies. For each parameter the proper bin size is calculated using ORIGIN 5.0. These plots are presented in following Fig. 2. From these histogram plots of galaxies it is seen that the distribution of observed numbers of galaxies are clearly inhomogeneous.

Figure 3 shows redshift distribution of the galaxy in the supercluster S [173+014+0082] [15]. In the map, low redshift galaxies are found in the substructure region. High redshifted galaxies are found to be distributed randomly [16]. The color map shows that the velocity dispersion is minimum in the region where the number density of the 
galaxy is minimum. In the substructure region, at $8^{0}$ declination, the redshift dispersion is found to be $\Delta \mathrm{z}=0.084$, whereas the lower substructure shows $\Delta \mathrm{z}=0.076$ and 0.080 , respectively. It seems that there is Gaussian distribution between redshift dispersion and number density of galaxies in the substructures. It shows that the Superclusters evolve naturally.

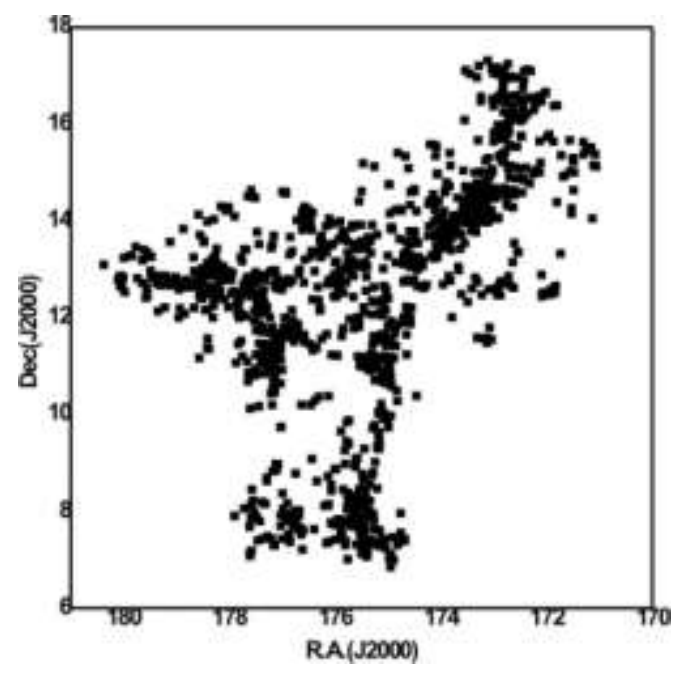

Fig. 1: All sky distribution of galaxies in the SDSS Supercluster $\mathrm{S}[173+014+0082]$ in equatorial coordinate system. Right ascension (R.A.) and Declination (Dec.) are given in degree.

In order to identify the preferred alignment of the spin vector of galaxies we have performed three different statistical tests [17]. For anisotropy, the limit of chi-square probability $\mathrm{P}\left(>\chi^{2}\right)$ is $<0.050$, auto correlation coefficient $(\mathrm{C} / \mathrm{C}(\sigma))$ is $>1.0$, first order Fourier coefficient $\left(\Delta_{11} / \sigma\left(\Delta_{11}\right)\right)$ is $>1.5$ and Fourier probability $\mathrm{P}\left(>\Delta_{11}\right)$ is $<0.150$ respectively.

For $\theta$ distribution (Table 1), the result of these tests is found as: the chi square probability $\mathrm{P}\left(>\chi^{2}\right)$ is 0.653 which is greater than critical value $(0.05)$ for anisotropy. In addition to this, the autocorrelation coefficient $\mathrm{C} / \mathrm{C}(\sigma)$ ) is 0.410 which is less than critical value; this result is in favor of isotropic distribution. Similarly, the first order Fourier coefficient $\left(\Delta_{11} / \sigma\left(\Delta_{11}\right)\right)$ and Fourier probability $\left(\mathrm{P}>\Delta_{1}\right)$ found to be 0.860 and 0.643 respectively. These two results also support the isotropic distribution of $\theta$. Hence, all three statistical tests show isotropic distribution of Supercluster S[173 + $014+0082]$ indicating random orientation of spin vectors of galaxies. From this result we can conclude that the galaxy formation model of Supercluster S $[173+014+$ 0082] follows the Hierarchy model. According to this model galaxies are believed to be formed from the clustering.

Fig. 4(a) shows the polar $(\theta)$ distribution of galaxies of the Supercluster S[173 + $014+0082]$. In the figure, solid and dotted curves represent expected isotropic and cosine distribution. In the small angle $\left(\theta<45^{\circ}\right)$ angle region i.e. shaded region no significant hump and dip are observed. While, in a larger angle $\left(\theta>45^{\circ}\right)$ a significant dip is observed at an angle $75^{\circ}$ with $1 \sigma$ error limit. In the shaded region the observed number of solutions is 1674 and expected number of solutions is 1652 . Thus the observed number of solutions exceeds the expected solution by 22 . This means that there are 11 more galaxies observed. On the other hand, in a larger angle region the observed solution is less than expected solution by 24 . This causes deficiency of 12 galaxies in that region. Furthermore, at the bimodal region one extra galaxy is observed. Thus these humps at small angle regions are totally nullified by the dip at the larger angle. The excess number of galaxies in the smaller angle can be regarded as transfer of the galaxies from the larger angle region. This may happen due to the gravitational tidal effect.

In $\phi$ distribution, the statistics (Table 1) support the isotropic distribution. The chi square probability ( $\left.\mathrm{P}>\chi^{2}\right)$ and auto-correlation coefficient $(\mathrm{C} / \mathrm{C}(\sigma)$ found to be 0.610 (greater than 0.05 ) and -0.481 (less than $1 \sigma$ ). Similarly, the value of first order Fourier coefficient $\left(\Delta_{11} / \sigma\left(\Delta_{11}\right)\right)$ and first order Fourier probability $\left(\mathrm{P}>\Delta_{1}\right)$ is -0.358 (less than $1.5 \sigma$ ) and 0.883 (greater than 0.15 ). These results are strong evidence to advocate the Hierarchy model of galaxy evolution in the Supercluster $\mathrm{S}[173+014+0082]$. 

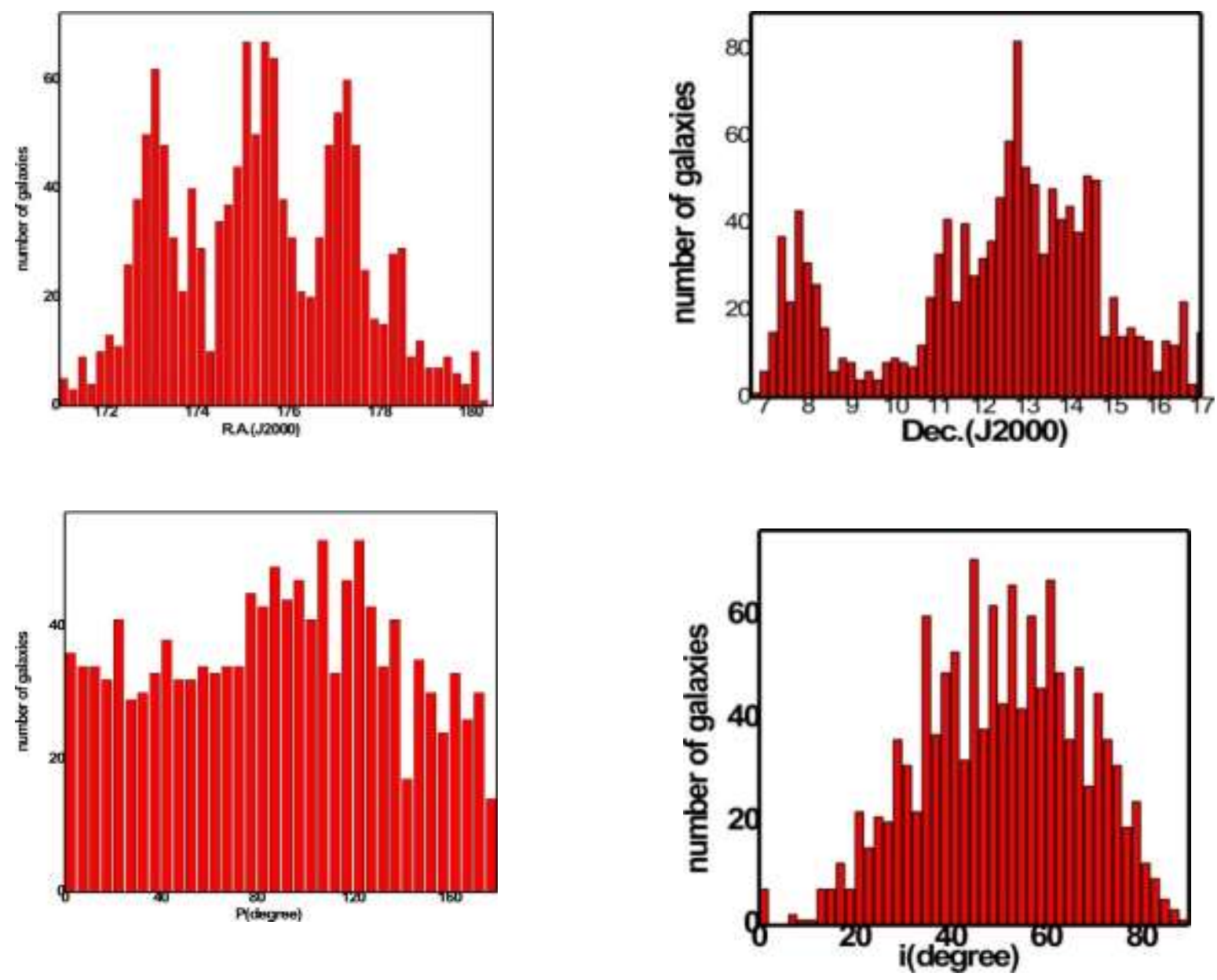

Fig. 2: The distribution of right ascension $(\alpha)$, declination $(\delta)$, position angle $(\mathrm{P})$ and inclination angle (i) of galaxies in the Supercluster S $[173+014+0082]$. The Y-axis represents the number of observed galaxies. The inhomogeneous distribution of galaxies can be seen.
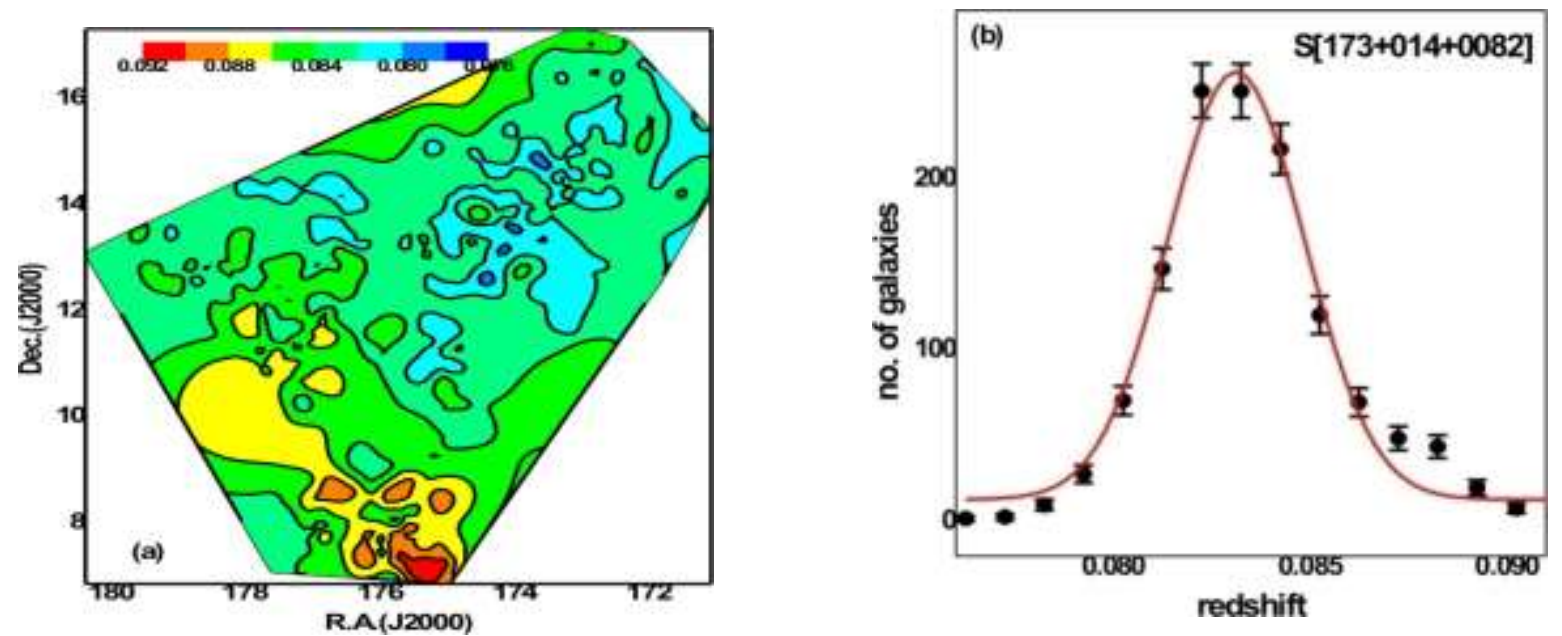

Fig. 3: (a) red shift map and (b) the distribution of galaxies in the Supercluster region. Solid curves represent Gaussian fit. The statistical error bars are shown. 
Fig. 4(b) represents the distribution of azimuthal angle of Supercluster S[173 + $014+0082]$. The solid and dotted line indicate the expected and average distribution. The observed number of solutions in the small angle region $\left(-90^{\circ}<\phi<-45^{0}\right)$ is 19 more than expected solution; however, no significant humps are observed in the distribution curve. Similar result is obtained at a larger angle region $\left(45^{0}<\phi<90^{\circ}\right)$ also. The shaded region represents the bimodal region $\left(-45^{0}<\phi<45^{\circ}\right)$. In this region the expected and observed numbers of solutions are 1405 and 1396 respectively. From the figure, two crystal clear humps are observed at $-5^{0}$ and $5^{\circ}$ and dip is observed at $-15^{\circ}$ within error limit $1 \sigma$.

Table 1: Statistics of the polar and azimuthal angle distributions of galaxies in the Supercluster $\mathrm{S}[173+014+0082]$. The first column represents the statistics used, $\mathrm{P}\left(>\chi^{2}\right)$ represents the chi-square probability (second row). Similarly, $\mathrm{C} / \mathrm{C}(\sigma)$ represents the auto-correlation coefficient(third row). The last two rows give the first order Fourier coefficient $\left(\Delta_{11} / \sigma\left(\Delta_{11}\right)\right)$ and first order Fourier probability $\mathrm{P}\left(>\Delta_{1}\right)$.

\begin{tabular}{|l|c|c|}
\hline \multicolumn{1}{|c|}{ Statistics } & $\begin{array}{c}\text { Polar angle } \\
(\boldsymbol{\theta})\end{array}$ & $\begin{array}{c}\text { Azimuthal angle } \\
(\phi)\end{array}$ \\
\hline $\mathrm{P}\left(>\chi^{2}\right)$ & 0.653 & 0.610 \\
\hline $\mathrm{C} / \mathrm{C}(\sigma)$ & 0.410 & -0.481 \\
\hline$\left(\Delta_{11} / \sigma\left(\Delta_{11}\right)\right)$ & 0.860 & -0.358 \\
\hline $\mathrm{P}\left(>\Delta_{1}\right)$. & 0.643 & 0.883 \\
\hline
\end{tabular}

\section{Conclusions}

The Supercluster S[173+014+0082] consists of 1302galaxies of which position angles, right ascension, declination, and axial ratio are known. These are obtained from two dimensional projections on the celestial sphere by SDSS DR 12 Survey. The conversion of two dimensional observed parameters into three dimensional is carried out by using 'position angleinclination' method. In order to find out the theoretical distribution of galaxy rotation axes we ran random simulation by generating $10^{7}$ virtual galaxies as

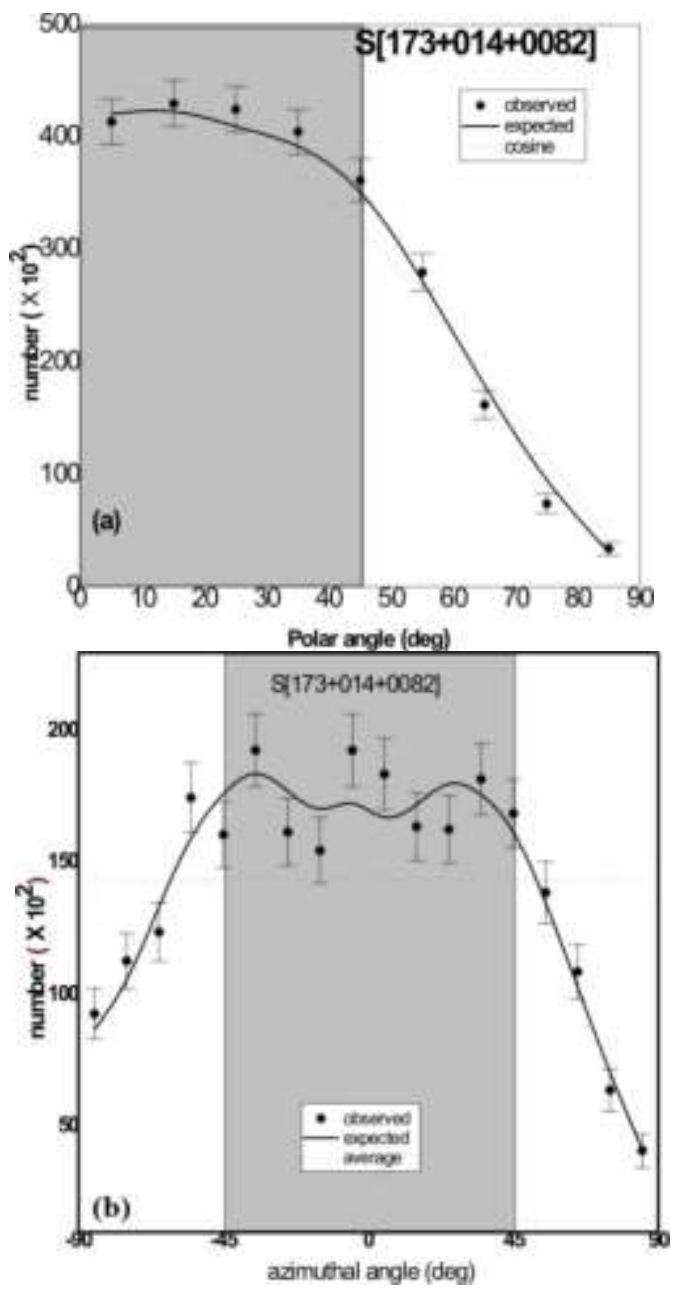

Fig. 4: (a) The polar $(\theta)$ and (b) azimuthal $(\varphi)$ angle distributions of galaxies in the Supercluster S[173+014+0082]. The solid line represents the expected isotropic distributions. The dash lines represent the cosine and average distributions respectively. The solid circles with $\pm 1 \square$ error bars are represent the observed distribution. proposed by Aryal \& Saurer. Here we assumed cosmological principle: distribution of galaxies in the large scale structure is homogeneous and isotropic. The simulated results were compared with the observed distribution using the statistical tools namely chi-square test, auto-correlation test and Fourier test. The distribution of spin vector and spin vector projections of total SDSS galaxies that have redshift in the range 0.076 to 0.091 are found to be random in all samples. We conclude our results as follows: 
(a) The polar and azimuthal angles of supercluster galaxies shows isotropic isotropic distribution suggesting no preferred alignment of angular momentum vectors as well as its projection. In other words we observed vanishing angular momentum in the Supercluster. This result advocates the Hierarchy model of galaxy evolution as suggested by Peebles.

(b) The redshift distribution of galaxies in Supercluster showed Gaussian-like nature, suggesting the distribution of galaxies' Hubble recessional velocity is isotropic, satisfying cosmological principle.

\section{Acknowledgements}

One of the authors (J.R.Malla) is thankful to the Department of Astro-Particle Physics, Innsbruck University, Austria, for providing data access . One of them (J.R.Malla ) is grateful to the authorities of the SDSS for providing the database.

\section{References}

[1] S. Colids, Jindal A Jindal., N. E. chisari, D. Vibert, Y. Dubois, C. Pichon, J. Devriendt. Galaxy Orientation with the cosmic web across cosmic time, Monthly Notice of the Royal Astronomical Society 481(2018) 4753-4774. https://doi.org/10.1093/mnras/sty2567.

[2] L. J. Liivamagi, E.Tempel and E.Saar, SDSS DR7 superclusters, Astronomy and Astrophysics 539 (2012) 1-14. https://doi.org/10.1051/0004-6361/201016288

[3] G. Abell, The distribution of rich clusters of galaxies, Astrophysical Journal Supplement 3 (1958) 211.

[4] D. G York, J. Adelman, J. E Anderson, Scott F. Anderson, James Annis, Neta A. Bahcallet.al. The sloan digital sky survey: Technical Summary. The Astronomical journal 120 (2000) 1579-1587.

[5] C. F. Weizscker, The new picture of the universe, Astrophysical Journal 114 (1951) 165.

[6] G. Gamow, The role of turbulence in the evolution of the Universe, Phys. Rev. 86 (1952) 251.

https://doi.org/10.1103/phys Rev.88.251.

[7] P. J. E. Peebles, Origin of the angular momentum of Galaxies, The Astrophysical J.155 (1969) 393.

[8] B. Aryal, S.N. Yadav \& W. Saurer , Spatial orientation of galaxies in the Zone of Avoidance. Bulletin of the Astronomical Society of India 40 (2012) 65.

[9] S. Djorgovski, Nearly normal galaxies, edited by S. M .Faber, Springer (1987)

[10] A. G. Doroshkevich, S. F. Shandarin, E. Saar, Spatial structure of protoclusters and the formation of Galaxies, Monthly Notices Royal Astron. Soc. 184 (1978) 643 - 660. https://doi.org/10.1093/mnras/184.3.

[11] L. M .Ozernoy, Sov.Astron, Whirl theory of the Origin and clusters of galaxiex, 15 (1972) 923.

[12] E. Holmberg, On the apparent diameters and the orientation in space of extragalactic Nebulae, Medd. Lund. Astron. Obs. 117 (1946) 3-82.

[13] P. Flin, W. Godlowski, The orientation of galaxy groups and formation of the local Supercluster, Monthly Notices Royal Astron. Soc. 222 (1986) 525.

[14] B. Aryal, W. Saurer, Comments on the expected isotropic distribution curvesin the galaxyorientation studies, Astronom. Astrophys. lett. 364 (2000) L97-L 100 .

http://articles.adsabs.harvard.edu/pdf/2000A\%26 A..364L..97A.

[15] C. M. casey ,D.Narayanan, A.Cooray, Dusty starforming galaxies at high redshift, Physics Reports Elsevier science Direct. 541 (2014) 45-161.

[16] S. N Yadav, B. Aryal, A study of r-and uMagnitude Dependence in the spatial Orientation of spin vectors of SDSS Galaxies having redshift $0.10<\mathrm{z}<0.11$. Himalayan Physics 5 (2015) 1 11. https://doi.org/10.3126/hj.v5io.12814.

[17] S. N Yadav, B. Aryal, W. Saurer, Preferred alignments of angular momentum vectors of six galaxies in six dynamically unstable Abell clusters. Research in Astronomy and Astrophysics, $\quad 17 \quad$ (2017) https://doi.org/10.1088/1674-4527/17/7/64 